\title{
Quando o tratamento se torna causa da doença
}

Jaime Oliveira', Fabiana Peixoto², Joana Silva Monteiro ${ }^{3-4}$

\section{RESUMO}

Introdução: A cefaleia é uma queixa frequente, não sendo raros os casos de automedicação. Este artigo relata a abordagem da cefaleia por uso excessivo de medicamentos (CUEM) numa doente automedicada com analgésicos, cuja intervenção foi dificultada pela má adesão ao acompanhamento médico.

Descrição do Caso: Mulher de 41 anos, empregada de limpeza. Antecedentes de enxaqueca desde a adolescência, agravada depois do segundo parto, com episódios semanais, sem alívio após tentativa de vários fármacos. Em 2015, após referenciação para consulta de neurologia, iniciou profilaxia com amitriptilina, tendo suspendido precocemente e abandonado o seguimento. Nos três anos seguintes teve consultas motivadas por doença aguda na unidade de saúde familiar, sem acompanhamento regular. Por persistência da enxaqueca recorreu ao serviço de urgência em jan/2018, onde foi referenciada novamente à consulta de neurologia, constatando-se automedicação com paracetamol + codeína, vinte supositórios em cada episódio de enxaqueca (com duração de três dias). Iniciou profilaxia com topiramato e agendou-se nova consulta. Suspendeu topiramato por emagrecimento e faltou à consulta. Recorreu à consulta da médica de família (MF) em jan/2019, referindo tristeza, insónia, obstipação e emagrecimento. Mantinha os episódios semanais de enxaqueca e o uso de supositórios. Após despiste de causa orgânica diagnosticou-se perturbação depressiva e CUEM. Explicou-se que o abuso medicamentoso poderia causar cefaleia e que o tratamento passaria pela cessação do fármaco. Foi medicada com antidepressivos e, nas consultas de reavaliação, constatou-se recuperação ponderal, melhoria do humor, cefaleia menos frequente e redução do uso de supositórios até à abstinência.

Comentário: A CUEM desenvolve-se pelo uso excessivo de medicação para a cefaleia, sendo a suspensão da medicação o único tratamento eficaz. A doente manteve uma situação de abuso medicamentoso durante oito meses, tendo como consequência uma CUEM e correndo risco de toxicidade hepática. O MF é fulcral na deteção e abordagem precoce do abuso medicamentoso.

Palavras-chave: Cefaleia; Enxaqueca; Analgésicos; Abuso medicamentoso; Cefaleia por uso excessivo de medicamentos.

\section{INTRODUÇÃO}

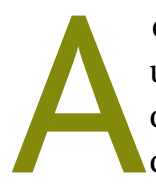
cefaleia é uma queixa prevalente e constitue uma das dez principais causas de incapacidade a nível mundial. ${ }^{1}$ A enxaqueca é o tipo de cefaleia que mais frequentemente leva o utente a procurar assistência médica. ${ }^{2}$ Um dos objetivos principais do tratamento adequado de qualquer cefaleia passa pela profilaxia da cefaleia por uso excessivo de medicamentos (CUEM). ${ }^{1,3}$

\footnotetext{
1. Médico Interno de Medicina Geral e Familiar. USF Odisseia, ACeS Grande Porto III - Maia/Valongo. Maia, Portugal.

2. Médica Assistente de Medicina Geral e Familiar. USF Pedras Rubras, ACeS Grande Porto III - Maia/Valongo. Maia, Portugal.

3. Médica Assistente de Medicina Geral e Familiar. USF Odisseia, ACeS Grande Porto III - Maia/Valongo. Maia, Portugal.

4. Professora Assistente. Faculdade de Medicina, Universidade do Porto. Porto, Portugal.
}

A enxaqueca é mais frequente nas mulheres e pode ter início em qualquer idade, com pico na adolescência/adulto jovem. ${ }^{1,3}$ Este tipo de cefaleia é precedido por aura num terço dos casos e, habitualmente, ocorre sob a forma de episódios que duram entre quatro a 72 horas. ${ }^{1,3}$ As características típicas da enxaqueca são as seguintes: unilateralidade, pulsatilidade, intensidade moderada ou grave, agravamento pela atividade física, associação com náuseas, fotofobia e fonofobia. ${ }^{1,3}$ Nas crises leves/moderadas, os agentes de primeira linha no tratamento sintomático são os anti-inflamatórios não esteroides (AINE) em associação com um antiemético. ${ }^{1,4-5}$ Quando o tratamento com AINE falha estão recomendados os triptanos, que constituem o tratamento de eleição para as crises moderadas/graves., ${ }^{1,4-5}$ Há indicação para o tratamento profilático perante crises com impacto na qualidade de vida do doente ou 
com interferência nas atividades de vida diária. ${ }^{1,4-5} \mathrm{~A}$ profilaxia diminui a frequência da cefaleia em mais de $50 \%$ e, para este efeito, medicamentos pertencentes à classe dos bloqueadores beta, bloqueadores dos canais de cálcio, modificadores do eixo renina angiotensina, antidepressivos ou antiepiléticos são opções válidas. ${ }^{1,4-5}$

Este artigo relata a abordagem da CUEM numa doente automedicada com analgésicos, cuja intervenção foi dificultada pela má adesão ao acompanhamento médico.

\section{DESCRIÇÃO DO CASO}

Apresenta-se o caso de uma mulher de 41 anos, caucasiana, com quatro anos de escolaridade e cuja profissão é empregada de limpeza. Trata-se de uma utente divorciada, a residir na Maia com o atual companheiro e com os seus dois filhos (sexo feminino, 19 anos, fruto do primeiro relacionamento; sexo masculino, sete anos, fruto do segundo relacionamento). A família da utente é do tipo «reconstruída» e enquadra-se na classe IV da classificação de Graffar.

Em termos de antecedentes pessoais refira-se: apendicectomia aos 11 anos; enxaqueca sem aura desde a menarca (aos 14 anos); tabagismo (12,5 UMA); 2Gesta2Para (eutócicos); varizes dos membros inferiores. A doente não tinha medicação crónica nem alergias medicamentosas conhecidas. Os antecedentes familiares da doente são apresentados na Figura 1.

De acordo com os registos clínicos disponíveis, a utente recorreu aos cuidados de saúde devido à enxaqueca por 27 vezes, entre 2009 e 2018. Em sete dessas ocasiões dirigiu-se à consulta aberta na sua unidade de saúde familiar (USF) e, nas restantes, ao serviço de urgência do hospital da área de influência.

Tipicamente, a utente queixava-se de cefaleia unilateral, pulsátil, muito intensa (intensidade 8-10/10, de acordo com a escala numérica da dor), agravada pela atividade física, associada a náuseas (por vezes com vómitos) e fonofotofobia, referindo necessidade de repousar num lugar sossegado e com pouca luz. Negava outros sintomas visuais ou sensitivos. Referia que estes episódios duravam dois a três dias e que costumava têlos duas vezes por mês, nos primeiros dias do cataménio e na semana anterior. Em nenhuma das avaliações realizadas apresentou alterações ao exame neurológi- co. Assim, de acordo com a The International Classification of Headache Disorders, a doente cumpria critérios de diagnóstico de enxaqueca sem aura. ${ }^{3}$

Após o nascimento do segundo filho, a utente passou a ter episódios de enxaqueca todas as semanas. Além disso, referia insónia inicial e intermédia, com sono pouco reparador. Nessa altura foi-lhe diagnosticada uma perturbação depressiva, para a qual foi medicada. Contudo, quer nesta altura quer posteriormente, a utente sempre teve um cumprimento errático das terapêuticas que lhe foram propostas de modo a tentar melhorar a sintomatologia depressiva.

Atendendo às queixas de enxaqueca foram prescritos vários analgésicos de forma sequencial, todos eles sem alívio sintomático, nomeadamente: ibuprofeno 600mg; diclofenac 100mg; acetilsalicilato de lisina $1000 \mathrm{mg}$; ácido acetilsalicílico $1000 \mathrm{mg}$; paracetamol $1000 \mathrm{mg}$; paracetamol + cafeína 500mg + 65mg; paracetamol + cafeína + tartarato de ergotamina + alcaloides da beladona $400 \mathrm{mg}+100 \mathrm{mg}+1 \mathrm{mg}+0,1 \mathrm{mg}$; tramadol + paracetamol 37,5mg + 325mg; paracetamol + fosfato de codeína + cloridrato de buclizina 500mg $+8 \mathrm{mg}+6,25 \mathrm{mg}$; zolmitriptano $5 \mathrm{mg}$.

Atendendo ao insucesso terapêutico, bem como à frequência e intensidade da enxaqueca, a doente foi referenciada para a consulta de neurologia, na qual foi avaliada em abril de 2015. Nessa consulta constatou-se que a utente apresentava perturbação depressiva e, associadamente, tinha pelo menos três episódios de enxaqueca por mês, duradouros e intensos, com fraca resposta à medicação sintomática. Perante o quadro, o neurologista assistente instituiu profilaxia farmacológica, optando pela amitriptilina 10mg ao deitar (com esquema de aumento progressivo da dose em cada semana, até $25 \mathrm{mg}$ ). Foi explicado à doente o intuito desta medicação e dada indicação para suspender toda a restante medicação sintomática, tendo sido medicada com naproxeno 500mg em SOS, até ao máximo de duas vezes por dia, em dois dias por semana. Segundo a utente, a amitriptilina surtiu efeito durante as primeiras duas semanas, com posterior perda do efeito, associado a agravamento da insónia. A utente suspendeu a toma quer da amitriptilina quer do naproxeno e faltou à consulta de neurologia subsequente.

Nos três anos seguintes, a utente recorreu à unidade de saúde apenas em situações de doença aguda, nas 


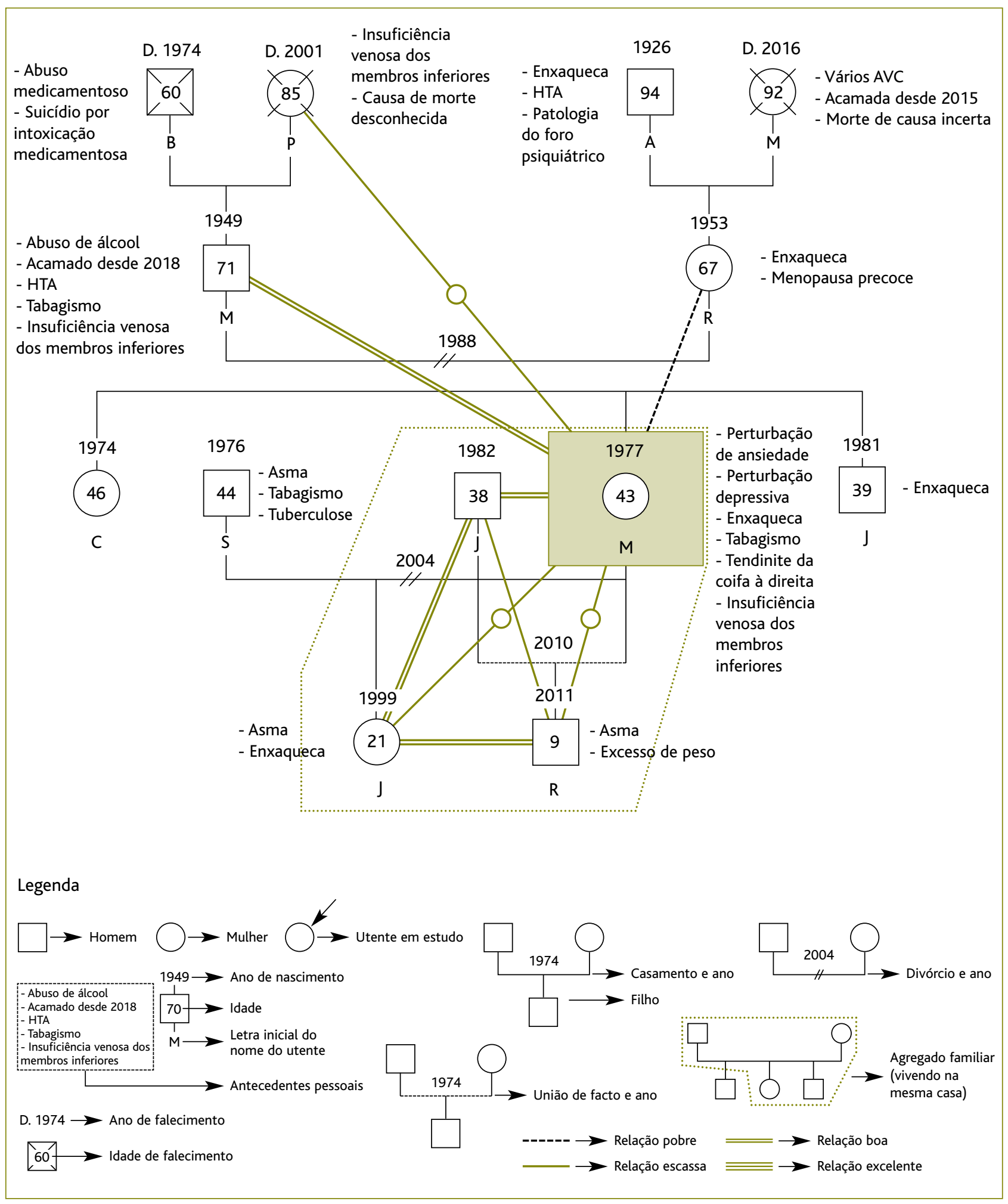

Figura 1. Genograma e Psicofigura de Mitchell, realizados com recurso ao Software GenoPro ${ }^{\circledR}$. 
quais foi atendida por vários médicos, sem seguimento regular pela médica de família.

A utente mantinha crises frequentes de enxaqueca e, em janeiro de 2018, recorreu ao serviço de urgência hospitalar onde realizou tomografia computadorizada crânio-encefálica, que não revelou qualquer alteração. No serviço de urgência foi referenciada novamente à consulta de neurologia, agendada para maio de 2018. Nesta consulta constatou-se que, por iniciativa própria, a utente fazia cerca de vinte supositórios de paracetamol + codeína 1000mg + 60mg durante três dias em cada episódio de enxaqueca. A utente referia que só conseguia controlar a enxaqueca com os supositórios, já que não tolerava a toma da medicação sintomática por via oral devido aos vómitos. No exame neurológico sumário não foram detetadas alterações identificáveis. O neurologista assistente decidiu iniciar profilaxia da enxaqueca com topiramato $25 \mathrm{mg}$ (com esquema de aumento progressivo da dose até $100 \mathrm{mg}$ ) e a doente ficou com consulta de reavaliação agendada para novembro de 2018, com indicação de levar preenchido um calendário de cefaleias. Contudo, a utente faltou à consulta de reavaliação.

Já em 2019, a utente recorreu à consulta aberta da sua médica de família, no dia 22 de janeiro, referindo humor depressivo, cansaço, insónia, aumento dos hábitos tabágicos, obstipação e perda ponderal de $7 \mathrm{~kg}$ face ao seu peso habitual (objetivamente apresentava $45 \mathrm{~kg}$ e um índice de massa corporal de $17,6 \mathrm{~kg} / \mathrm{m}^{2}$ ). Além disso, mantinha episódios de enxaqueca todas as semanas, durando cerca de três dias, e continuava a usar paracetamol + codeína para alívio sintomático, estando naquela altura a usar cerca de dez supositórios por dia. Tinha suspendido o topiramato por iniciativa própria, por não ter achado que tivesse benefício e porque leu na bula que este fármaco poderia causar perda ponderal.

Nesta consulta pediu-se estudo analítico e endoscópico de modo a despistar, por um lado, uma causa orgânica para o emagrecimento e, por outro, alterações da função hepática. Interpretou-se este quadro como uma cefaleia mista, incluindo enxaqueca e CUEM, associada a uma perturbação depressiva. Explicou-se à utente que o fármaco usado para alívio da cefaleia desde maio de 2018 era, pelo menos em parte, a causa da própria cefaleia e que o único tratamento eficaz seria a suspensão do medicamento que usava excessivamen- te. Iniciou-se escitalopram 10mg e mirtazapina 15mg. Além disso, deu-se indicação à doente para suspender gradualmente a toma dos supositórios e para fazer naproxeno, associado a metoclopramida se necessário, como tratamento "de ponte", usado no máximo em dois dias por semana, visando o alívio sintomático durante a retirada da medicação.

A médica de família explicou à utente que, inicialmente, a cefaleia poderia acentuar-se e poderiam surgir náuseas, vómitos, ansiedade e insónia. Para que este período de transição fosse melhor tolerado atribuiu-se à utente um certificado de incapacidade temporária, agendou-se consulta de reavaliação dentro de duas semanas e, até lá, garantiu-se à utente acessibilidade para poder ser consultada em caso de sintomas intoleráveis.

Alertou-se que seria necessário estabelecer uma aliança terapêutica entre a equipa de saúde familiar e a utente, com o compromisso de um seguimento mais regular durante o processo de cessação da medicação que era alvo de abuso. Finalmente, explicou-se que seria importante o preenchimento de um calendário de cefaleias para monitorizar a evolução das queixas. A utente concordou com o plano proposto.

A doente compareceu à consulta agendada no dia 11 de fevereiro. Nessa altura apresentava estabilidade ponderal e estava a fazer três supositórios de paracetamol + codeína 1000mg + 60mg por dia, quando tinha crises. Reforçou-se a importância de continuar a redução do consumo desta medicação e agendou-se nova reavaliação.

A utente foi reavaliada no dia 21 de fevereiro. Nesta altura referiu que já não fazia paracetamol + codeína $1000 \mathrm{mg}+60 \mathrm{mg}$ desde há três dias. Trazia os resultados das análises e da endoscopia e da colonoscopia realizadas, sendo que nenhum dos exames apresentava alterações de relevo.

Em consulta, no dia 13 de março, constatou-se estabilidade ponderal e a abstinência de paracetamol + codeína 1000mg $+60 \mathrm{mg}$. A doente referia melhoria importante do humor e da insónia, bem como uma diminuição do número de episódios de cefaleia. Contudo, não tinha, até essa data, preenchido o calendário de cefaleias. Reforçou-se a importância de manter a abstinência e de preencher o calendário.

Atendendo ao facto de ser uma grande frequentadora da consulta dos cuidados de saúde primários por 


\begin{tabular}{|l|l|l|l|l|}
\hline Nascimento do filho, R & $\begin{array}{l}\text { Problemas conjugais } \\
\text { Conflito com a irmã, } \\
\text { levando a corte da } \\
\text { relação afetiva }\end{array}$ & $\begin{array}{l}\text { Melhoria da situação } \\
\text { conjugal }\end{array}$ & $\begin{array}{l}\text { Foi vítima de acidente de viação. Por este motivo, deixou de } \\
\text { ser capaz de exercer as funções de cuidadora do pai. } \\
\text { O seu pai ficou permanentemente alectuado. } \\
\text { Conflito com o irmão, levando a corte da relação afetiva. } \\
\text { Ficou desempregada. }\end{array}$ \\
\hline Biografia & $\begin{array}{l}2011-2014 \\
2011\end{array}$ & 2014 & $2018-2019$ \\
\hline Patografia & $\begin{array}{l}\text { Diagnóstico de } \\
\text { perturbação depressiva } \\
\text { e insónia }\end{array}$ & $\begin{array}{l}\text { Melhoria dos sintomas } \\
\text { depressivos } \\
\text { Agravamento da } \\
\text { enxaqueca }\end{array}$ & $\begin{array}{l}\text { Paralisia facial central direita } \\
\text { Tendinite da coifa à direita } \\
\text { Aumento do tabagismo } \\
\text { (1 maço/dia) }\end{array}$ & $\begin{array}{l}\text { Agravamentos dos sintomas } \\
\text { depressivos } \\
\text { Abuso medicamentoso } \\
\text { Agravamento da enxaqueca } \\
\text { Diagnóstico de CUEM }\end{array}$ \\
\hline
\end{tabular}

Figura 2. Linha de vida de Medalie, desde 2011.

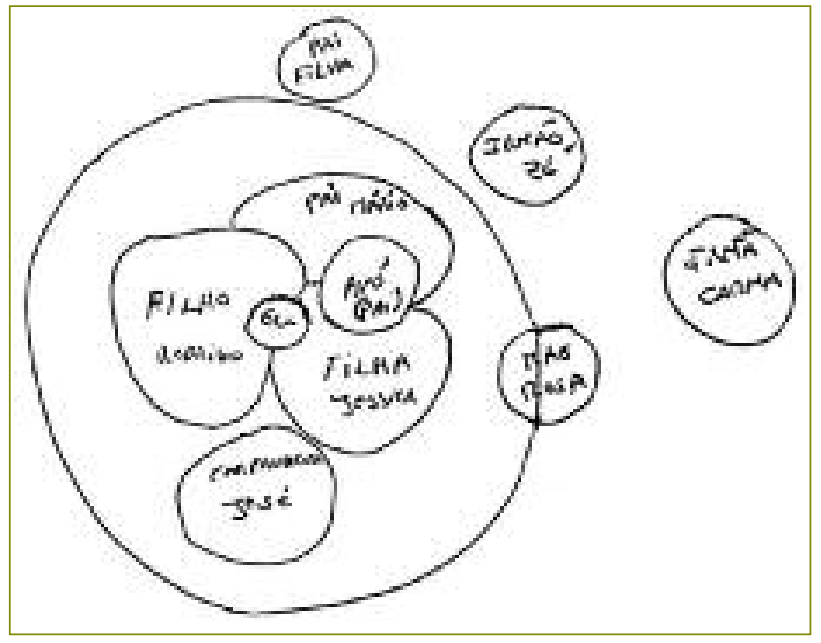

Figura 3. Círculo Familiar de Thrower. A utente referia estar descontente com a representação que a própria fez, pelos seguintes motivos: gostava que a sua avó paterna estivesse viva e perto de si; gostava de ter uma relação mais próxima com os irmãos e com a mãe; apesar de se constatar uma família altamente funcional de acordo com o APGAR familiar de Smilkstein (tinha 7 pontos em 9 possíveis), a utente referia que não recorreria a ninguém no caso de precisar de ajuda. Este isolamento social poderia explicar-se, em parte, pela perturbação depressiva.

situações agudas, sem seguimento regular em consulta programada, e ao facto de se ter identificado um abuso medicamentoso com consequências importantes para a saúde da própria, realizou-se nesta consulta a avaliação familiar da utente. Parte dos resultados dessa avaliação podem ser consultados nas Figuras 1, 2 e 3.
Por um lado, no genograma (Figura 1) é evidente uma perigosa combinação de antecedentes, nomeadamente o abuso de substâncias (álcool, tabaco e fármacos) na linhagem paterna e a enxaqueca na linhagem materna. Por outro lado, a linha de vida de Medalie (Figura 2) denota uma associação entre vários acontecimentos na vida da utente e o surgimento ou agravamento de patologias. Finalmente, o círculo familiar de Thrower que a utente construiu (Figura 3) representa os conflitos familiares e o isolamento social vividos pela utente, ambos em íntima relação com a perturbação depressiva.

Nas consultas subsequentes da utente, realizadas ao longo do ano de 2019, constatou-se melhoria progressiva do quadro clínico. Na última avaliação da utente, em 21 de fevereiro de 2020, registou-se a manutenção da abstinência, recuperação ponderal (pesava $54 \mathrm{~kg}$ e tinha um índice de massa corporal de $\left.20,3 \mathrm{~kg} / \mathrm{m}^{2}\right)$, normalização dos hábitos tabágicos, melhoria do humor e uma menor frequência de episódios de enxaqueca. Segundo o calendário de cefaleias, nesta fase apresentava episódios quinzenais de enxaqueca, com duração inferior a três dias e com pouca interferência no dia-a-dia, pelo que se manteve a terapêutica previamente instituída.

\section{COMENTÁRIO}

A CUEM desenvolve-se devido ao uso excessivo de medicação para a cefaleia, sendo o único tratamento eficaz a suspensão da medicação em causa. ${ }^{1,3-4}$ É mais 
comum em mulheres e tem como fatores de risco as perturbações depressivas e de ansiedade.

Este caso permite refletir sobre as dificuldades que acarreta o seguimento dos utentes que, por um lado, têm uma adesão irregular às consultas programadas, mas que, por outro lado, são hiperfrequentadores das consultas destinadas à resolução de patologia aguda. Perante este tipo de situações reitera-se a importância de aplicar ferramentas de avaliação familiar.

A avaliação poderá ser útil para tentar compreender eventos de vida associados a este tipo de conduta e, se possível, cessar este comportamento, privilegiando um acompanhamento programado e regular pelo médico de família, focado na prevenção.

Por outro lado, as técnicas de comunicação e avaliação familiar ajudam a perceber o padrão expectável de certas doenças numa dada família. ${ }^{6}$ Neste caso constata-se uma perigosa combinação de antecedentes, nomeadamente o abuso de substâncias (álcool, tabaco e fármacos) na linhagem paterna e a enxaqueca na linhagem materna, percetível na Figura 1.

Perante os antecedentes familiares apresentados desaconselha-se a prescrição de fármacos com potencial para o desenvolvimento de consumo abusivo e de dependência, como os opioides e os fármacos contendo cafeína.

$\mathrm{O}$ abuso medicamentoso também parece ter sido potenciado pela perturbação do humor apresentada pela doente. Esta perturbação foi sucessivamente agravada pelos eventos de vida desestruturantes pelos quais a utente passou desde 2011, como é evidenciado na Figura 2. Atendendo a este facto, a utente poderia beneficiar da avaliação quer por psiquiatria, quer por psicologia, visando a otimização da terapêutica farmacológica e não farmacológica e promovendo o desenvolvimento de mecanismos de coping face a eventos de vida adversos.

A utente apresentada manteve uma situação de abuso medicamentoso durante cerca de oito meses, tendo como consequência uma CUEM e correndo risco de toxicidade hepática. Com este relato de caso pretende-se, por um lado, alertar para a existência de situações semelhantes à apresentada e, por outro, enaltecer o papel da medicina geral e familiar na deteção e abordagem precoce quer das perturbações do humor, quer das situações de abuso medicamentoso.
No caso particular da CUEM existem vários aspetos a ter em conta. Quando o médico prescreve terapêutica de alívio sintomático para a cefaleia deve alertar o utente para a possibilidade de se poder desenvolver uma CUEM e, deste modo, promover a sua profilaxia através da educação para a saúde. Além disso, na abordagem terapêutica da CUEM é importante assegurar a vigilância clínica apertada e o seguimento regular do utente por parte do médico de família, o que neste caso em particular poderá ter contribuído para o sucesso na intervenção. Ainda assim, é importante que, perante o insucesso terapêutico desta estratégia inicial, alguns casos poderão necessitar de referenciação para consulta hospitalar de abordagem de dependências.

\section{REFERÊNCIAS BIBLIOGRÁFICAS}

1. European Headache Federation. Princípios europeus da abordagem das cefaleias comuns nos cuidados de saúde primários [homepage]. Lisboa: Sociedade Portuguesa de Cefaleias; 2010. Available from: http://www. cefaleias-spc.com/comunidade/documentos/

2. Wootton RJ,Wippold II FJ, Whealy MA. Evaluation of headache in adults. UpToDate [Internet]; 2020 [updated 2021 Aug 12; cited 2020 Feb 10]. Available from: https://www.uptodate.com/contents/evaluation-ofheadache-in-adults

3. Headache Classification Committee of the International Headache Society (IHS). The international classification of headache disorders, 3rd ed. Cephalalgia. 2018;38(1):1-211.

4. Becker WJ, Findlay T, Moga C, Scott NA, Harstall C, Taenzer P. Guideline for primary care management of headache in adults. Can Fam Physician. 2015;61(8):670-9.

5. Grupo de Estudio de Cefaleas de la SEN. Guías diagnósticas y terapéuticas de la Sociedad Española de Neurología 2015. Madrid: Sociedad Española de Neurología; 2015. ISBN 9788415198994

6. Rebelo L. A família em medicina geral e familiar: conceitos e práticas. Coimbra: Almedina; 2018. ISBN 9789724073132

\section{CONFLITO DE INTERESSES}

Os autores declaram não possuir quaisquer conflitos de interesse.

\section{FINANCIAMENTO}

Os autores declaram que este relato de caso não foi objeto de qualquer tipo de financiamento.

\section{ENDEREÇO PARA CORRESPONDÊNCIA \\ Jaime Oliveira \\ E-mail: jaimelroliveira@gmail.com \\ https://orcid.org/0000-0002-2424-2392}

Recebido em 25-05-2020

Aceite para publicação em 03-06-2021 


\section{ABSTRACT}

\section{WHEN TREATMENT BECOMES THE CAUSE OF THE DISEASE}

Introduction: Headache is a common complaint and self-medication is not uncommon. This article reports the approach to medication overuse headache $(\mathrm{MOH})$ in a patient who self-medicated with analgesics, whose intervention was hampered by poor adherence to medical follow-up.

Case Description: 41-year-old woman, cleaning employee. History of migraine since adolescence, which worsened after her second delivery, presenting with weekly episodes without relief after several treatments were prescribed. In 2015, after referral to a Neurology consultation, she started prophylaxis with amitriptyline, which she suspended, missing the follow-up appointment. In the three years that followed, she only attended primary care consultations motivated by acute illness, without regular appointments. On Jan/2018, she went to the emergency department due to persistent migraine, and again she was referred to a Neurology consultation, where it was detected that she self-medicated with over twenty suppositories of paracetamol + codeine in each episode of migraine. Topiramate was prescribed and a new consultation was scheduled. She suspended topiramate due to weight loss and missed the follow-up appointment. On Jan/2020, she consulted her family doctor reporting sadness, insomnia, constipation, and weight loss. She maintained the migraine and the use of suppositories. After screening for organic causes, she was diagnosed with depressive disorder and $\mathrm{MOH}$. It was explained that drug abuse could cause headaches and treatment would involve the cessation of that medication. She was medicated with antidepressants, and, in the next consultations, weight recovery, improved mood, less frequent headache, and reduced suppository use until withdrawal were documented.

Comment: $\mathrm{MOH}$ develops due to the excessive use of headache relievers, and the suspension of medication is the only effective treatment. This patient maintained a situation of medication overuse for eight months, resulting in $\mathrm{MOH}$ and risk of liver toxicity. The family doctor is crucial to early detecting and addressing drug abuse.

Keywords: Headache; Migraine; Analgesics; Drug abuse; Medication overuse headache. 